\title{
Absence of Thermophoretic Flow in Relativistic Heavy-Ion Collisions as an Indicator for the Absence of a Mixed Phase
}

\author{
Markus H. Thoma \\ Max-Planck-Institut für extraterrestrische Physik, Giessenbachstraße, 85748 Garching, Germany
}

(November 5, 2018)

\begin{abstract}
If a quark-gluon plasma is formed in relativistic heavy-ion collisions, there may or may not be a mixed phase of quarks, gluons and hadronic clusters when the critical temperature is reached in the expansion of the fireball. If there is a temperature gradient in the fireball, the hadronic clusters, embedded in the heat bath of quarks and gluons, would be subjected to a thermophoretic force. It is shown that, even for small temperature gradients and short lifetimes of the mixed phase, thermophoresis would lead to a flow essentially stronger than the observed one. The absence of this strong flow provides support for a rapid or sudden hadronization mechanism without a mixed phase.
\end{abstract}

PACS numbers: 25.75.-q, 25.75.Ld, 12.38.Mh

Relativistic heavy-ion collision experiments at CERN (SPS, LHC) and BNL (RHIC) are dedicated to the discovery of the quark-gluon plasma, a hypothetic state of matter in which quarks and gluons are deconfined. The basic idea is to create a hot and dense fireball in a high-energy nucleus-nucleus collision with a temperature above the critical one of the order $T_{c}=170 \mathrm{MeV}$, predicted by QCD lattice computations [1]. This tiny fireball will rapidly expand and reach the transition temperature after a few $\mathrm{fm} / \mathrm{c}$. Depending on the order of the deconfinement phase transition there might be a mixed phase of quarks, gluons and hadrons. QCD lattice simulations of the deconfinement phase transition show either a continuous (cross over) or a first order phase transition depending crucially on the masses of the dynamical quarks [1].

Although the question of the order of the phase transition is still open [1], let us assume that there is a first order phase transition from the quark-gluon plasma to the hadronic matter leading to a mixed phase. Possible consequences of a mixed phase in relativistic heavy-ion collisions have been extensively studied (see e.g. Ref. [2]). Here we want to discuss a new aspect of the mixed phase, namely the possibility of a thermophoretic flow.

Since hadronization does not take place instantaneously - although hadronziation may proceed rapidly [3] - the hadronic bubbles might contain confined clusters of quarks instead of the final hadrons. These clusters or pre-hadrons as well as usual hadrons are heavy and extended objects embedded in the thermal quarkgluon plasma phase similar as dust particles in a lowtemperature plasma [4].

It is reasonable to assume that the temperature of the fireball is higher at the center than at the surface of the fireball. For example, in hydrodynamical calculations a local maximum temperature differing from the average temperature by as much as $70 \mathrm{MeV}$ has been taken to explain photon data at SPS [5]. Hence, we presuppose the existence of a temperature gradient in the fireball. For simplicity, we assume a constant temperature gradient from the center to the surface of a spherical fireball. Owing to this temperature gradient in the thermal heat bath of the quark-gluon plasma a thermophoretic force will act on the hadronic clusters similar as on dust particles in a low-temperature plasma [6]. This force will push the hadrons from the center to the surface resulting in a radial outward motion of hadrons as sketched in Fig.1. Here we assumed furthermore that the hadronic bubbles are not transparent for the thermal partons but that elastic scattering of the partons off the pre-hadrons is dominant.

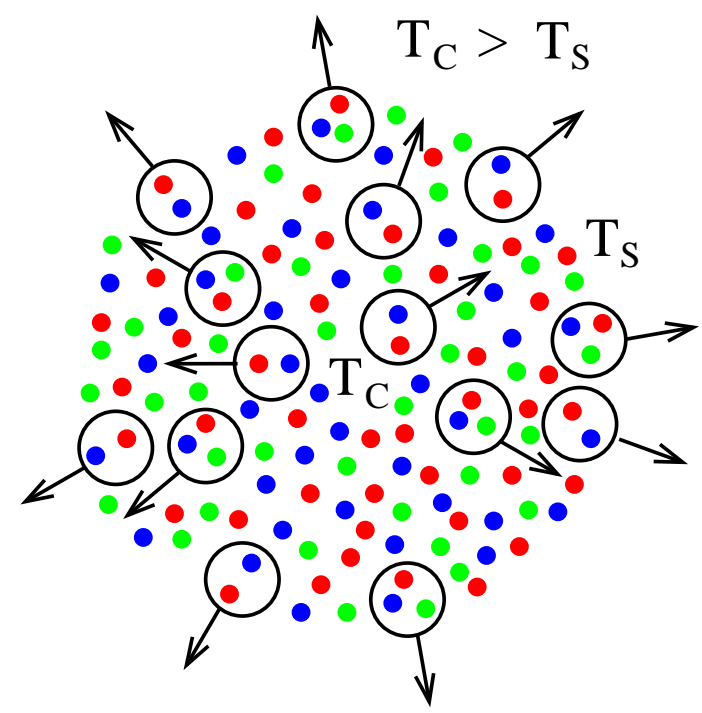

FIG. 1. Sketch of thermophoresis in the mixed phase of the fireball in relativistic heavy-ion collisions acting on hadrons. The small dots are the partons which are the thermal background for the hadrons or quark clusters indicated by the circles.

A temperature gradient in the mixed phase, for which the temperature is given by the critical temperature, could be realized in two different ways. First, a finite chemical potential increasing from the center to the surface would imply a decreasing critical temperature. Second, if there is a constant chemical potential, a tempera- 
ture gradient in the fireball leads to a mixed-phase shell moving outward. Due to the finite mean free path the partons from the hotter inner regions can interact with the hadrons in the mixed-phase shell. These possibilities could be considered using hydrodynamical methods [7].

The collective motion of hadrons, called flow, is an important feature of heavy-ion collisions which has been investigated theoretically and experimentally in great detail, providing important informations on the equation of state of the fireball and especially a possible signature for the quark-gluon plasma formation [8]. The different flow patterns (e.g. elliptic and radial flow), studied so far, are caused by a pressure gradient in the fireball. Here we propose a new source for flow, namely a temperature gradient in the fireball. This directed, collective motion of hadrons superimposes the usual hydrodynamical flow.

Using elementary kinetic arguments, the thermophoretic force can be estimated similar as the heat conductivity [9]. The latter follows from the energy flux in the presence of a temperature gradient. Instead of the energy transfer we consider the momentum transfer per unit time and area in a relativistic plasma. Then the thermophoretic force $F$ per area $A$ is given as

$$
\frac{F}{A}=-\frac{1}{3} n\langle u\rangle \lambda \frac{d\langle p\rangle}{d T} \frac{d T}{d r}
$$

where $n$ is the number density of the quark-gluon plasma, $\langle u\rangle \simeq 1$ the thermal velocity of the partons, $\lambda$ their mean free path, and $\langle p\rangle \simeq 3 T$ their average momentum.

Hence, the thermophoretic force acting on a hadron is given by

$$
F=-\pi n \lambda R^{2} \frac{d T}{d r}
$$

where $R$ is the hadron radius. Using typical values for the mean free path and the density of the partons at $T=T_{c}, \lambda=1 \mathrm{fm}, n=5 \mathrm{fm}^{-3}$, and for the hadron radius, $R=1 \mathrm{fm}$, and assuming, for example, a small temperature gradient of $d T / d r=1 \mathrm{MeV} / \mathrm{fm}$, we get $F=$ $-16 \mathrm{MeV} / \mathrm{fm}$.

Now, we want to calculate the final velocity $v_{f}$ of a hadron with mass $m$. Using

$$
-F=\frac{d}{d t} \frac{m v}{\sqrt{1-v^{2}}}=\frac{d}{d x} \frac{m v^{2}}{\sqrt{1-v^{2}}}
$$

we find

$$
W=-F d=\frac{m v_{f}^{2}}{\sqrt{1-v_{f}^{2}}}
$$

with the fireball radius $d$. Solving this equation for $v_{f}$ yields

$$
v_{f}=\frac{W}{\sqrt{2} m}\left(\sqrt{1+\frac{4 m^{2}}{W^{2}}}-1\right)^{1 / 2}
$$

For pions $(m=140 \mathrm{MeV})$ and $d=10 \mathrm{fm}$ we obtain $v_{f}=0.81$ and for protons $(m=940 \mathrm{MeV}) v_{f}=0.40$, i.e. a strong flow larger or at least comparable to the hydrodynamic flow as extracted from two-particle correlation measurements (Hanbury Brown/Twiss interferometry), which yield typically $v_{f} \simeq 0.5[10]$. The thermophoretic force, being proportional to $R^{2}$, and consequently the flow would have been even stronger if we had assumed a larger radius $R$ for the hadronic clusters. Note also that the thermophoretic flow velocity is smaller for massive hadrons than for lighter ones.

Here we assumed that the mixed phase lives for 10 $20 \mathrm{fm} / \mathrm{c}$ for accelerating the hadrons over the distance $d$ from the center to the surface of the fireball. Such a long lifetime of the mixed phase would occur only for a strong first order transition as predicted by simple hydrodynamical models using an unrealistic equation of state (see e.g. [11]). According to lattice results, showing at most a weak first order transition, this assumption is probably a crude overestimation. However, even for small lifetimes of the mixed phase of $1-2 \mathrm{fm} / \mathrm{c}$, corresponding to $d \simeq 1$ $\mathrm{fm}$, we obtain the same result for the final velocities if we take a moderate temperature gradient of $10 \mathrm{MeV} / \mathrm{fm}$. This follows from the fact that $W$ is proportional to $d$ times $d T / d r$.

For our purpose the use of elementary kinetic theory, generalized here to relativistic plasmas, is justified since a more elaborate treatment of thermophoresis based on the Boltzmann equation (Enskog-Chapman method) 12 differs only by $20 \%$ from the elementary kinetic result in the non-relativistic limit [6]. Similarly, the viscosity of the quark-gluon plasma has been estimated from elementary kinetic arguments [13] in good agreement with a transport theoretical approach 14.

We have seen that the thermophoretic force on hadrons in the mixed phase, caused by a small to moderate temperature gradient, leads to a strong flow in addition to the hydrodynamical flow. This result is in contrast to present observations at SPS [15]. Also the thermophoretic flow velocity is larger for lighter than for heavier hadrons, which contradicts experimental results showing a higher flow velocity for baryons than for mesons. We take this as an indication for the absence of a mixed phase in relativistic heavy-ion collisions, investigated so far, i.e. either a continuous or second order phase transition or no phase transition at all, also in aggreement with a rapid hadronization scenario [3]. Anyway, the possible presence of a temperature gradient in a heavy-ion fireball could have other consequences which could be an interesting subject for future hydrodynamical investigations, e.g. including dissipative effects such as heat conductivity.

\section{ACKNOWLEDGMENTS}

The author would like to thank U. Heinz, P. Huovinen, 
and H. Rothermel for helpful discussions.

[1] F. Karsch, hep-lat/0109017.

[2] D.H. Rischke and M. Gyulassy, Nucl. Phys. A608, 479 (1996).

[3] see e.g. L.P. Csernai and I.N. Mishustin, Phys. Rev. Lett. 74, 5005 (1995) and references therein.

[4] A. Bouchoule (Ed.), Dusty Plasmas (John Wiley, 1999).

[5] P. Huovinen, P.V. Ruuskanen, and S.S. Räsänen, nuclth/011105.

[6] H. Rothermel, T. Hagl, G. Morfill, and H.M. Thomas, physics/0110045.

[7] P. Huovinen, private communication.

[8] J.-Y. Ollitrault, Nucl. Phys. A638, 195c (1998).

[9] F. Reif, Fundamentals of Statistical and Thermal Physics (McGraw-Hill, 1965).

[10] U. Heinz, Nucl. Phys. A661, 140c (1999).

[11] F.D. Steffen and M.H. Thoma, Phys. Lett. B 510, 98 (2001).

[12] L. Waldmann, Z. Naturf. A 14, 590 (1959).

[13] M.H. Thoma, Phys. Lett. B 269, 144 (1991).

[14] G. Baym, H. Monien, C.J. Pethick, and D.G. Ravenhall, Phys. Rev. Lett. 64, 1867 (1990).

[15] J.M. Burward-Hoy and B.V. Jacak, nucl-ex/0111005. 